\title{
Acute effects of apple extracts with different polyphenol compositions on glucose transport in human intestinal cell line Caco-2/TC7
}

\author{
Emily Prpa, Christopher Corpe and Wendy Hall \\ King's College London, London, United Kingdom
}

\section{Abstract}

Diets with a high-glycaemic load may increase the risk of type 2 diabetes. Food and beverages containing large amounts of highly bioaccessible starch and sugars induce pronounced postprandial blood glucose concentrations. Chronic exposure to sharp blood glucose peaks can lead to oxidative stress and pancreatic beta-cell dysfunction. Fruit polyphenols may help to limit the glucose excursion following a high-carbohydrate meal.

Our previous research showed that $1.8 \mathrm{~g}$ polyphenol-rich apple extract (AE) mixed into a drink consumed before a high starch + sucrose meal, inhibited the incremental area under the curve of plasma glucose during the early absorption-driven postprandial phase $(0-30 \mathrm{~min})$ by $52 \%$ relative to a placebo drink and meal. Our latest dose-response trial showed that the glycaemic response to starch and sucrose was modified by $0.9 \mathrm{~g}$ AE. Possible mechanisms include activity inhibition of carbohydrate digestive enzymes and/or glucose absorption (SGLT1/GLUT2 transport). The AE is uniquely high in phlorizin (10.6\%) - a strong inhibitor of SGLT1. However, evidence shows that other apple polyphenols may also inhibit SGLT1.

The primary aim of our work was to explore the effect of different mixtures of apple polyphenols on gut transporters involved in glucose uptake. Acute dose-response effect of the AE and three fractions of AE $(20 \%, 40 \%$ and $80 \%$ methanol elution during fractionation) on total glucose transport were investigated in Caco-2/TC7 cells. Uptake media (10min) contained, except for controls, increasing concentrations of AE/fractions $(0-4.5 \mathrm{mg} / \mathrm{ml})$ incorporating the physiological range estimated to be present in the small intestine after ingestion of clinical trial test drinks. Glucose transport under sodium-dependent conditions was determined by radiochemical detection of Glucose D-[14C(U)] and Glucose L-[1-14C].A 47\% decrease in total glucose uptake was observed with 1.12mg/ $\mathrm{ml} \mathrm{AE} \mathrm{(i.e.} \mathrm{clinical} \mathrm{trial} \mathrm{highest} \mathrm{physiological} \mathrm{dose)} \mathrm{v} \mathrm{control}(P=0.03)$. Dose-response experiments showed $\mathrm{IC}_{50} \mathrm{were}: 1.29 \mathrm{mg} / \mathrm{ml}$, $0.31 \mathrm{mg} / \mathrm{ml}$ and $0.88 \mathrm{mg} / \mathrm{ml}$ for AE (mg/g: 79 phlorizin; 26 epicatechin; 4 quercetin), 80\% fraction (mg/g: $101 \mathrm{phlorizin;} 26 \mathrm{epicatechin;}$ 38 quercetin), 40\% fraction (mg/g: 83 phlorizin; 20 epicatechin; 7 quercetin), respectively. The $20 \%$ fraction (mg/g: 4 phlorizin; 12 epicatechin; 2 quercetin) had no effect on glucose transport.

We have gained greater understanding of a potential mechanism for the anti-hyperglycaemic properties of apple polyphenols, supporting potential applications of the AE in functional foods/beverages. Our in vitro data suggest that a combination of higher amounts of phlorizin and quercetin may be important to maximise intestinal glucose uptake inhibition.

\section{Conflict of Interest}

Funded by DIANA Food Ltd 